\title{
In Vitro Antifungal Activity of CAN-296: A Naturally Occurring Complex Carbohydrate
}

\author{
Avital Mazar Ben-Josef ${ }^{\dagger} *$, Elias K. Manavathu ${ }^{\dagger}$, David Platt $^{\dagger \dagger}$ \\ and JACK D. SOBEL ${ }^{\dagger}$ \\ ${ }^{\dagger}$ Division of Infectious Diseases, Department of Medicine, \\ School of Medicine, Wayne State University, \\ Detroit, MI, U.S.A. \\ ${ }^{\dagger+}$ IGG International Inc. \\ Cambridge, MA, U.S.A.
}

(Received for publication May 26, 1997)

\begin{abstract}
The in vitro activity of a naturally occurring complex carbohydrate, CAN-296, was evaluated by testing 132 clinical and ATCC isolates of yeast and Aspergillus fumigatus, many of which were azole-resistant. The in vitro susceptibility tests were performed by standardized broth microand macrodilution methods and results were compared with those obtained for amphotericin B, fluconazole, ketoconazole, flucytosine and the pneumocandin L-733,560. All tested Candida species showed highly uniform susceptibility to CAN-296 at concentrations of 0.078 to $0.312 \mu \mathrm{g} / \mathrm{ml}$; non-albicans Candida were as susceptible to CAN-296 as the Candida albicans strains. Multi-azoleresistant Candida species were highly sensitive to CAN-296. Minimum inhibitory concentration measurements did not differ from minimum lethal concentrations by more than two-fold for all tested Candida species. Aspergillus fumigatus, on the other hand, showed only moderate susceptibility to CAN-296. The kinetics of the anti-Candida activity of CAN-296 was investigated by killcurve experiments using $C$. albicans and $C$. glabrata and the results were compared with those obtain for amphotericin B. CAN-296 was found to be rapidly fungicidal in concentrations ranging from $4 \sim 16$ fold the mean MIC value. The broad spectrum of anti-Candida activity together with the rapid fungicidal effect make this complex carbohydrate a promising agent for clinical use.
\end{abstract}

The incidence of fungal infections increased dramatically in the past decade. This is due, in part, to an expanding population of immunocompromised patients (from cytotoxic therapy, steroid therapy and AIDS) and technical advances that facilitate fungal invasion. The increased frequency, severity, and number of fungal species identified as pathogens has further created a critical need for new, safe anti-fungal drugs. Today, the most commonly used drugs for systemic and local fungal infections are amphotericin B (AMB) and azole agents ${ }^{13}$. $A M B$ is still the drug of choice in many systemic mycoses due to its broad spectrum and fungicidal activity, however, AMB is nephrotoxic and is administered intravenously only ${ }^{2}$. The triazoles, fluconazole and itraconazole have broad spectrum of activity, can be administered orally, and are less toxic than AMB. However, azoles are fungistatic and resistance has become a significant problem ${ }^{3 \sim 8)}$. Primary and secondary resistance to flucytosine is common and this drug is generally used only in combination with AMB.

In this study we report on the in vitro antifungal effects of CAN-296, a new, naturally occurring, complex carbohydrate isolated from the cell wall of the fungus Mucor rouxii. Although its molecular structure has not been completely elucidated yet, CAN-296 is known to consist of terminal and branched $N$-acetyl-D-glucosamine. It has a molecular mass of 4300 daltons.

\section{Materials and Methods}

We tested the activity of CAN-296 against 132 clinical and ATCC isolates of yeast and Aspergillus fumigatus, including azole-resistant strains. The results were compared with the antifungal activity of azoles, AMB, flucytosine and L-733,560 pneumocandin ${ }^{6)}$.

\section{Organisms and Culture Conditions}

Yeasts: A total of 112 strains from 14 yeast species were tested. Most of the strains $(90 \%)$ were clinical isolates; the rest were obtained from the American Type Culture Collection (Rockville, MD). All isolates had been stored in Litmus Milk (Becton Dickinson Microbiology Systems, Cockeysville, MD), and kept frozen in $-70^{\circ} \mathrm{C}$. For use, approximately $10 \mu \mathrm{l}$ of the stock culture was plated onto Sabouraud dextrose agar (SDA) and incubated for 48 hours, and then a colony was transferred to another SDA plate and incubated for 24 hours. The latter served as the source of the inoculum for most of the studies. About $30 \%$ of the tested isolates were azoles-resistant (fluconazole 48 hours MIC $>32 \mu \mathrm{g} / \mathrm{ml}$ ). 
The distribution of species included 29 Candida albicans (14 azole-resistant), 15 Candida glabrata (5 azoleresistant), 10 Candida parapsilosis (2 azole-resistant), 10 Candida lusitaniae; 2 Candida rugosa; 10 Candida tropicalis (2 azole-resistant), 1 azole-resistant Candida lambica; 12 azole-resistant Candida krusei; 10 Candida guillermondii; 5 Candida kefyr; 4 Candida stellatoidea; 1 Candida paratropicalis; 1 Candida lipolitica and 2 Saccharomyces cerevisiae.

Aspergillus: Twenty Aspergillus fumigatus [10 clinical isolates (Microbiology Laboratory, Detroit Medical Center, Detroit, Michigan), 5 laboratory isolates each resistant to itraconazole and amphotericin B] strains were used to study the effect of CAN-296 on growth of Aspergillus species. Stock cultures were prepared using conidia as inoculum on YPD (Sigma Chemical Company, St. Louis, MO) agar slants. The slants were incubated at $30^{\circ} \mathrm{C}$ for 4 days, or until the cultures conidiated, and fresh conidia from the subcultures were used as the source of inoculum for all the cultures in subsequent work. The subculturing enabled us to assess further the purity of the original cultures.

\section{CAN-296}

CAN-296 is a naturally occurring heat stable complex carbohydrate isolated from the cell wall of the fungus Mucor rouxii. Structurally, CAN-296 belongs to a novel class of antifungal agents. It consists mostly of 1,4-, 3,4-and 4,6-linked, and terminal $N$-acetyl-D-glucosamine residues. It has a molecular mass of 4300 daltons. The compound was obtained as a $0.1 \mathrm{mg} / \mathrm{ml}$ aqueous solution from IGG International Inc., Cambridge, MA.

\section{Yeast Antifungal Susceptibility Assays}

The minimum inhibitory concentration (MIC) and minimum lethal concentration (MLC) of CAN-296 were determined using the broth microdilution method as recommended by the National Committee for Clinical Laboratory Standards (NCCLS) ${ }^{9}$ ) except Peptone Yeast Extract Glucose Medium (PYG) was used for the assay instead of RPMI 1640. Briefly, the microorganisms were grown in PYG medium to which CAN-296 was added to a final concentration of 10 to $0.019 \mu \mathrm{g} / \mathrm{ml}$. The MIC was defined as the lowest concentration that inhibited growth completely. The MICs were recorded after 48 hours of incubation at $35^{\circ} \mathrm{C}$. MLC was determined by subculturing $100 \mu \mathrm{l}$ from the first well demonstrating complete growth inhibition and from all wells that had no visible growth onto Sabouraud dextrose agar plates that were incubated at $30^{\circ} \mathrm{C}$ for 24 hours. MLC was defined as the lowest concentration at which $99 \%$ of the initial inoculum was killed.

\section{Aspergillus fumigatus Susceptibility Studies}

The susceptibility of $A$. fumigatus to CAN-296 as well as to itraconazole and amphotericin $\mathbf{B}$ was determined by the broth macrodilution technique according to the procedure of ESPINEL-INGROFF et al. ${ }^{10)}$ as modified by Manavathu et $a .^{11)}$ using fresh conidia as the source of inoculum. For the preparation of conidial suspensions, cultures of various isolates were grown on YPD agar plates for 6 days at $30^{\circ} \mathrm{C}$ until the whole plate was covered with fungal growth. The conidia were collected by flooding the agar surfaces with sterile growth medium $(20 \mathrm{ml})$ followed by gentle scraping with a sterile rubber policeman. The resulting suspension, containing fragments of mycelium, small pieces of agar, and other cellular debris, was collected by aspiration. The aspirate was vortexed vigorously to release conidia from the conidiophores and was filtered through a sterile cotton plug fitted in a sterile Nalgene filtration funnel. In general, a single filtration step was sufficient to obtain a clarified condial suspension. The cell density was determined by hemocytometer count. Each sample was counted four times independently, and the mean value of the quadruplicate was used for the calculation of the cell density. Under these condition we routinely obtained approximately $1 \sim 3 \times 10^{7}$ conidia per $\mathrm{ml}$ when $20 \mathrm{ml}$ of growth medium was used for resuspension. The relationship between cell density estimated by hemocytometer count and the number of colony forming units (CFU) was determined by plating appropriately diluted cell suspensions on YPD agar. The cell density estimation by CFU production was approximately 10 to 20 percent lower than the value obtained by hemocytometer count.

The broth macrodilution experiments were performed in sterile 6-ml polystyrene tubes (Falcon 2058) with a final volume of $1 \mathrm{ml}$. Two times the required final concentrations of CAN-296, itraconazole and AMB were prepared in $0.5 \mathrm{ml}$ growth medium by two-fold serial dilutions. Each well was inoculated with an equal volume $(0.5 \mathrm{ml})$ of conidial suspension $(2 \times$ the required final CFU prepared in growth medium by two-fold serial dilution) to obtain a final CFU of $1 \times 10^{4}$ per ml. Each series of drug concentration was tested in triplicate and each MIC determination was repeated at least once. The AMB tubes were wrapped in aluminum foil to prevent light exposure, and all tubes were incubated at $35^{\circ} \mathrm{C}$ for 48 hours, and scored for visible growth after vortexing the tubes gently, or scraping the walls of the tube fol- 
Table 1-1. In vitro susceptibilities of C. albicans and non-albicans Candida to CAN-296 in comparison to azoles, amphotericin B and L-733,560.

\begin{tabular}{|c|c|c|c|c|}
\hline Organism & Antifungal agent & $\begin{array}{c}\mathrm{MIC}(\mu \mathrm{g} / \mathrm{ml}) \\
\text { Range }\end{array}$ & $\begin{array}{c}\mathrm{MIC}(\mu \mathrm{g} / \mathrm{ml}) \\
\text { Mean }\end{array}$ & $\begin{array}{c}\operatorname{MLC}(\mu \mathrm{g} / \mathrm{ml}) \\
\text { Range }\end{array}$ \\
\hline \multirow{7}{*}{ C. albicans (29) } & CAN-296 & $0.156 \sim 0.312$ & 0.268 & $0.156 \sim 0.312$ \\
\hline & Amphotericin B & $0.02 \sim 0.1$ & 0.050 & \\
\hline & Flucytosine & $0.04 \sim 1.25$ & 0.330 & \\
\hline & Ketoconazole & $0.01 \sim 6.25$ & 0.282 & \\
\hline & Fluconazole & $0.08 \sim 80$ & 23.95 & \\
\hline & Itraconazole & $0.01 \sim 12.5$ & 0.660 & \\
\hline & L-733,560 & $0.05 \sim 0.78$ & 0.203 & \\
\hline \multirow[t]{7}{*}{ C. parapsilosis $(10)$} & CAN-296 & $0.078 \sim 0.312$ & 0.165 & $0.078 \sim 0.312$ \\
\hline & Amphotericin B & $0.05 \sim 0.2$ & 0.185 & \\
\hline & Flucytosine & $0.08 \sim 1.25$ & 0.321 & \\
\hline & Ketoconazole & $0.02 \sim 0.2$ & 0.030 & \\
\hline & Fluconazole & $0.16 \sim 20$ & 3.730 & \\
\hline & Itraconazole & $0.02 \sim 0.2$ & 0.072 & \\
\hline & $\mathrm{L}-733,560$ & $0.02 \sim 1.56$ & 0.157 & \\
\hline \multirow[t]{7}{*}{ C. glabrata (15) } & CAN-296 & $0.078 \sim 10.00$ & 0.812 & $0.078 \sim 10.00$ \\
\hline & Amphotericin B & $0.05 \sim 0.2$ & 0.115 & \\
\hline & Flucytosine & $0.04 \sim 0.3$ & 0.070 & \\
\hline & Ketoconazole & $0.01 \sim 6.3$ & 0.440 & \\
\hline & Fluconazole & $1.25 \sim 40$ & 16.53 & \\
\hline & Itraconazole & $0.02 \sim 6.3$ & 1.613 & \\
\hline & $L-733,560$ & $0.1 \sim 0.39$ & 0.300 & \\
\hline \multirow{7}{*}{ C. tropicalis (10) } & CAN-296 & $0.039 \sim 0.312$ & 0.195 & $0.039 \sim 0.312$ \\
\hline & Amphotericin B & $0.02 \sim 0.39$ & 0.176 & \\
\hline & Flucytosine & $0.08 \sim 0.63$ & 0.215 & \\
\hline & Ketoconazole & $0.02 \sim 3.12$ & 0.460 & \\
\hline & Fluconazole & $0.63 \sim 80$ & 27.56 & \\
\hline & Itraconazole & $0.02 \sim 6.25$ & 0.885 & \\
\hline & L-733,560 & $0.1 \sim 0.78$ & 0.308 & \\
\hline \multirow[t]{7}{*}{ C. lusitaniae (10) } & CAN-296 & $0.156 \sim 0.312$ & 0.265 & $0.156 \sim 0.312$ \\
\hline & Amphotericin B & 0.05 & 0.05 & \\
\hline & Flucytosine & 0.08 & 0.08 & \\
\hline & Ketoconazole & $0.02 \sim 0.2$ & 0.11 & \\
\hline & Fluconazole & $0.31 \sim 20$ & 7.186 & \\
\hline & Itraconazole & $0.02 \sim 0.1$ & 0.17 & \\
\hline & L-733,560 & $0.39 \sim 0.78$ & 0.624 & \\
\hline \multirow[t]{7}{*}{ C. krusei (12) } & CAN-296 & $0.039 \sim 0.312$ & 0.263 & $0.039 \sim 0.312$ \\
\hline & Amphotericin B & $0.1 \sim 0.39$ & 0.222 & \\
\hline & Flucytosine & $2.5 \sim 20$ & 11.56 & \\
\hline & Ketoconazole & $0.01 \sim 1.56$ & 0.611 & \\
\hline & Fluconazole & $0.16 \sim 80$ & 51.27 & \\
\hline & Itraconazole & $0.01 \sim 0.39$ & 0.227 & \\
\hline & $\mathrm{L}-733,560$ & 0.78 & 0.78 & \\
\hline \multirow[t]{7}{*}{ C. guillermondii (10) } & CAN-296 & $0.078 \sim 0.312$ & 0.171 & $0.078 \sim 0.312$ \\
\hline & Amphotericin B & $0.2 \sim 0.78$ & 0.392 & \\
\hline & Flucytosine & $0.08 \sim 0.16$ & 0.115 & \\
\hline & Ketoconazole & $0.02 \sim 0.1$ & 0.035 & \\
\hline & Fluconazole & $5 \sim 10$ & 5.70 & \\
\hline & Itraconazole & $0.2 \sim 0.78$ & 0.364 & \\
\hline & $\mathrm{L}-733,560$ & $0.78 \sim 1.56$ & 1.248 & \\
\hline \multirow[t]{7}{*}{ C. kefyr (5) } & CAN-296 & $0.156 \sim 0.312$ & 0.187 & $0.156 \sim 0.312$ \\
\hline & Amphotericin B & $0.05 \sim 0.39$ & 0.210 & \\
\hline & Flucytosine & $0.08 \sim 0.16$ & 0.1 & \\
\hline & Ketoconazole & 0.02 & 0.02 & \\
\hline & Fluconazole & $0.31 \sim 2.5$ & 1.64 & \\
\hline & Itraconazole & 0.2 & 0.2 & \\
\hline & $\mathrm{L}-733,560$ & $0.02 \sim 0.78$ & 0.295 & \\
\hline \multirow[t]{5}{*}{ C. stellatoidea (4) } & CAN-296 & 0.312 & 0.312 & 0.312 \\
\hline & Flucytosine & $0.625 \sim 1.25$ & 0.937 & \\
\hline & Ketoconazole & 0.01 & 0.01 & \\
\hline & Fluconazole & $0.16 \sim 0.3 \mathrm{I}$ & 0.235 & \\
\hline & Itraconazole & 0.01 & 0.01 & \\
\hline
\end{tabular}


Table 1-2. In vitro susceptibilities of non-albicans Candida to CAN-296 in comparison to azoles, amphotericin B and L-733,560.

\begin{tabular}{|c|c|c|c|c|}
\hline Organism & Antifungal agent & $\begin{array}{c}\mathrm{MIC}(\mu \mathrm{g} / \mathrm{ml}) \\
\text { Range }\end{array}$ & $\begin{array}{c}\text { MIC }(\mu \mathrm{g} / \mathrm{ml}) \\
\text { Mean }\end{array}$ & $\begin{array}{c}\mathrm{MLC}(\mu \mathrm{g} / \mathrm{ml}) \\
\text { Range }\end{array}$ \\
\hline \multirow[t]{5}{*}{ C. rugosa (2) } & CAN-296 & 0.312 & 0.312 & 0.312 \\
\hline & Flucytosine & 0.08 & 0.08 & \\
\hline & Ketoconazole & 0.01 & 0.01 & \\
\hline & Fluconazole & 5 & 5 & \\
\hline & Itraconazole & 0.02 & 0.02 & \\
\hline \multirow[t]{6}{*}{ C. lambica (1) } & CAN-296 & 0.078 & 0.078 & 0.078 \\
\hline & Amphotericin B & 0.01 & 0.01 & \\
\hline & Flucytosine & 0.31 & 0.31 & \\
\hline & Ketoconazole & 0.01 & 0.01 & \\
\hline & Fluconazole & 20 & 20 & \\
\hline & Itraconazole & 0.01 & 0.01 & \\
\hline C. paratropicalis $(\mathrm{I})$ & CAN-296 & 0.312 & 0.312 & 0.312 \\
\hline C. lipolitica (1) & CAN-296 & 0.312 & 0.312 & 0.312 \\
\hline Saccharomyces cerevisiae (2) & CAN-296 & 0.156 & 0.156 & 0.156 \\
\hline
\end{tabular}

lowed by vortexing. The MIC was defined as the lowest concentration of the drug in which no visible growth occurred. The medium recommended by NCCLS was RPMI 1640. However this media was unsuitable for studying the susceptibility of yeast to CAN-296 because this complex carbohydrate consistently precipitated in RPMI 1640, even at low concentration, whereas no precipitation occurred in PYG medium.

\section{Time-kill Study}

The anti-Candida activity of CAN-296 was examined by kill-curve experiments using Candida albicans isolate (B311), and Candida glabrata (32554). Briefly, test organisms were grown in YPG broth for 24 hours at $30^{\circ} \mathrm{C}$ on a gyratory shaker ( $\left.160 \mathrm{rpm}\right)$. The fresh 24 hours culture was diluted approximately 1000 -fold to obtain a cell density of $1 \times 10^{6} \mathrm{CFU}$ per $\mathrm{ml}$. Five $\mathrm{ml}$ aliquots of the diluted cultures were incubated at $30^{\circ} \mathrm{C}$ with various concentrations $(0 \sim 5 \mu \mathrm{g} / \mathrm{ml})$ of CAN-296. For comparison with $\mathrm{AMB}, 5 \mathrm{ml}$ aliquots of the diluted cultures were incubated at $30^{\circ} \mathrm{C}$ in the presence of 5 and $10 \mu \mathrm{g} / \mathrm{ml}$ of CAN-296 and 4 and $8 \mu \mathrm{g} / \mathrm{ml}$ of AMB. At various time intervals ( $0 \sim 24$ hours) $0.1 \mathrm{ml}$ aliquots of cell suspension were removed, serially diluted $\left(10^{2}\right.$ to $10^{6}$ fold $)$ and $0.1 \mathrm{ml}$ aliquots were spread on YPD agar plates in replicates. After incubation at $30^{\circ} \mathrm{C}$ for 48 hours, the number of CFU per $\mathrm{ml}$ of cultures were calculated and plotted against the time of exposure to CAN-296 to construct a kill-curve.

\section{Results}

Susceptibility of Yeasts to CAN-296

Table 1 provides a summary of the in vitro susceptibility studies. The vast majority $(99 \%)$ of the tested species showed highly uniform susceptibility to CAN-296 at concentration of 0.078 to $0.312 \mu \mathrm{g} / \mathrm{ml}$. Azole-resistant and azole-susceptible Candida species were equally sensitive, and when compared to L-733560, CAN-296 had a narrower and more consistent therapeutic range. The MICs of CAN-296 were comparable with those of AMB. One strain of Candida glabrata was relatively insensitive to CAN-296 (MIC $=10 \mu \mathrm{g} / \mathrm{ml})$. For all azolesusceptible and resistant Candida species, the MIC and MLC did not differ by more than two fold.

\section{Susceptibility of A. fumigatus to CAN-296}

The MIC values of CAN-296 for clinical and laboratory isolates of $A$. fumigatus were compared with those obtained for AMB and ITZ. Nineteen of the 20 isolates tested showed no inhibition of growth (MIC $>5 \mu \mathrm{g} / \mathrm{ml}$ ). The only exception was the clinical isolate W73355 which yielded an MIC value of $0.625 \mu \mathrm{g} / \mathrm{ml}$. Both itraconazoleresistant and amphotericin B-resistant aspergillus isolates, were as susceptible to CAN-296 as were the itraconazole- and amphotericin B-susceptible strains.

\section{Fungicidal Activity}

The time-dependent fungicidal activity of CAN-296 for Candida albicans and Candida glabrata was studied by kill curve experiments. The concentrations of CAN296 used for the time-kill study ranged from approximately $2 \sim 16$ fold the mean MIC value for most Candida 
species. As shown in Fig. 1-A, the fungicidal activity of CAN-296 was both concentration and time dependent. The fungicidal action was rapid and greater than $99 \%$ of the cells were killed within 15 minutes of exposure to the compound when CAN-296 concentration used was greater than 16 fold the MIC $(5 \mu \mathrm{g} / \mathrm{ml})$. Greater than $99 \%$ killing was achieved within 45 minutes at a concentration of $2.5 \mu \mathrm{g} / \mathrm{ml}$ and within 120 minutes at a concentration of $1.25 \mu \mathrm{g} / \mathrm{ml}$ (Fig. 1-A). Candida glabrata was shown to be slightly less susceptible than Candida albicans, however $95 \%$ killing was achieved within 120 minutes at a concentration of $5 \mu \mathrm{g} / \mathrm{ml}$ (Fig. 1-B).

The time-dependent fungicidal activity of CAN-296 against Candida albicans and Candida glabrata was notably expeditious when compared with that of AMB (Figs. 2 and 3). CAN-296 at $5 \mu \mathrm{g} / \mathrm{ml}$ and $10 \mu \mathrm{g} / \mathrm{ml}$ killed greater than $95 \%$ of the cells within 60 and 30 minutes of exposure to the drug, respectively. No significant difference in the rate of killing was observed between the two Candida species used. The fungicidal activity of AMB was not as rapid, with $95 \%$ kill achieved within 120 minutes at a concentration of $8 \mu \mathrm{g} / \mathrm{ml}$ and within 4 hours at a concentration of $4 \mu \mathrm{g} / \mathrm{ml}$. Moreover, C. glabrata was less susceptible to killing by AMB than $C$. albicans, at a concentration of $4 \mu \mathrm{g} / \mathrm{ml}$ considerably more than 4 hours of exposure was required to obtain $>95 \%$ killing (Fig. 3).
Fig. 1. Kill-curve study of CAN-296 at concentrations 2 16 fold MIC value against Candida albicans B311 (A) and Candida glabrata 32554 (B).

$0 \mu \mathrm{g} / \mathrm{ml}$

$1.25 \mu \mathrm{g} / \mathrm{ml}, \triangle 2.5 \mu \mathrm{g} / \mathrm{ml}$

$5 \mu \mathrm{g} / \mathrm{ml}$

A

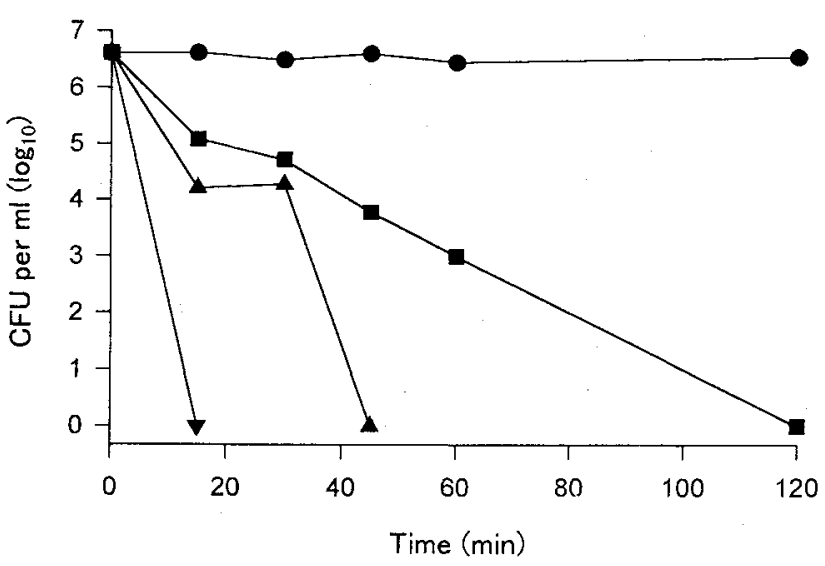

B

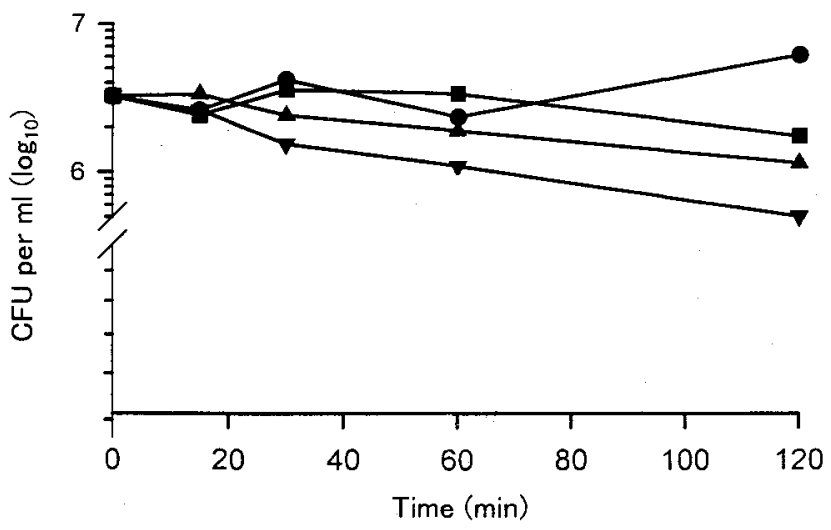

Fig. 2. Comparison of fungicidal activity of CAN-296 and amphotericin B-for C. albicans.

○ CAN-296; $5 \mu \mathrm{g} / \mathrm{ml}, \square \mathrm{CAN}-296 ; 10 \mu \mathrm{g} / \mathrm{ml}, \diamond \mathrm{AMB} ; 4 \mu \mathrm{g} / \mathrm{ml}, \triangle \mathrm{AMB} ; 8 \mu \mathrm{g} / \mathrm{ml}$.

Candida glabrata kill curve: comparison between CAN-296 and AMB

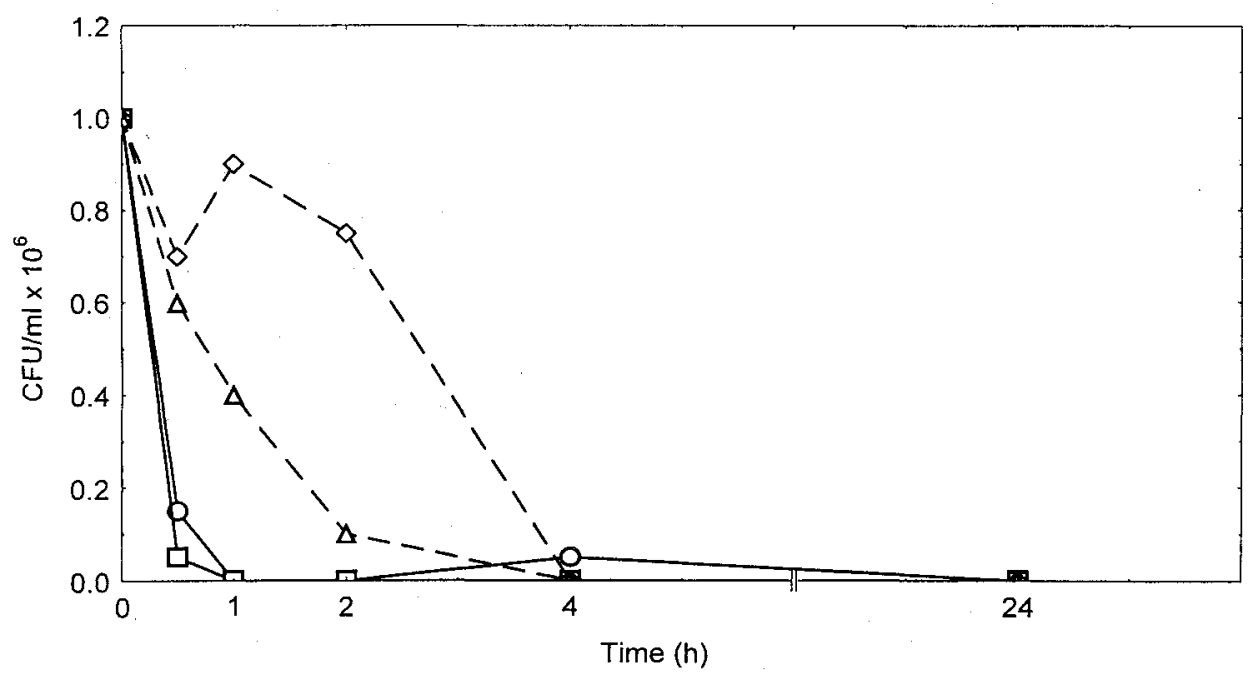


Fig. 3. Comparison of fungicidal activity of CAN-296 and amphotericin B-for C. glabrata.

○ CAN-296; $5 \mu \mathrm{g} / \mathrm{ml}, \square \mathrm{CAN}-296 ; 10 \mu \mathrm{g} / \mathrm{ml}, \diamond \mathrm{AMB} ; 4 \mu \mathrm{g} / \mathrm{ml}, \triangle \mathrm{AMB} ; 8 \mu \mathrm{g} / \mathrm{ml}$.

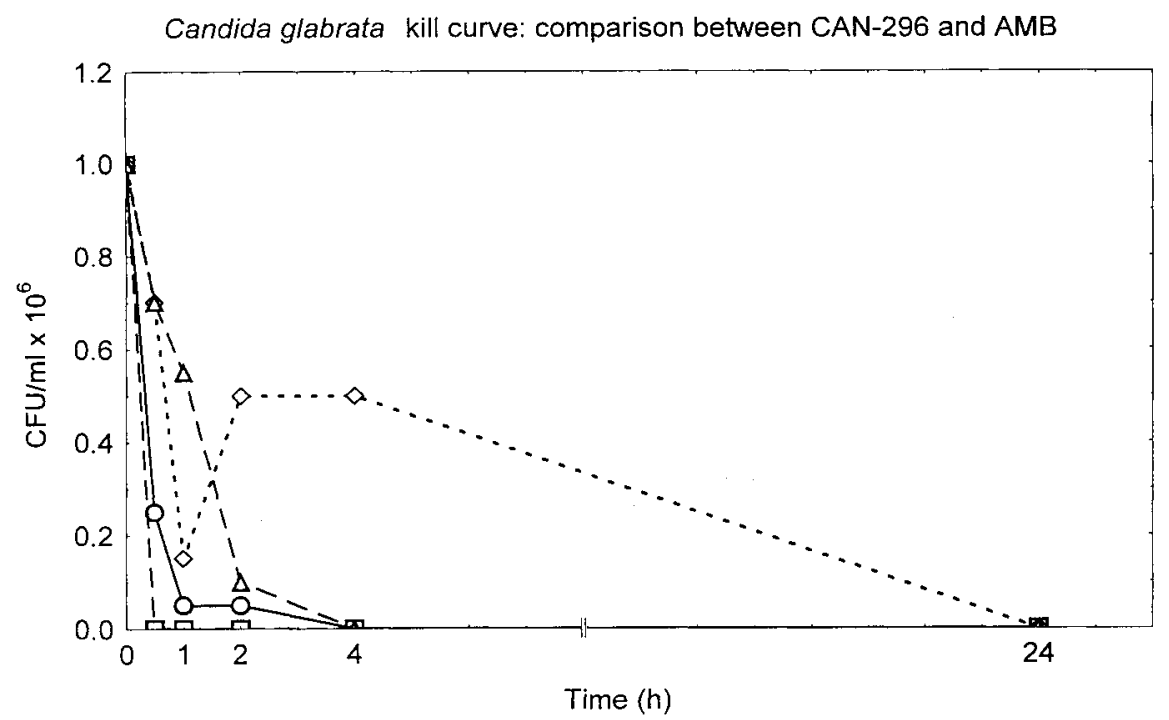

\section{Discussion}

The results of the present study demonstrate that CAN-296 is a highly active antifungal agent in vitro. Its broad anti-Candida activity is comparable to that of AMB. It possesses a very narrow therapeutic range $(0.078 \sim 0.312 \mu \mathrm{g} / \mathrm{ml})$ in all Candida species tested. The non-albicans Candida species were as susceptible as the Candida albicans strains. MICs did not differ between the azole-susceptible and the azole-resistant Candida species. When compared with L-733,560, CAN-296 was found to possess uniform activity against all the tested Candida species; in contrast L-733,560 demonstrated a much wider therapeutic range $0.02 \sim 1.56 \mu \mathrm{g} / \mathrm{ml}$ with the same species tested (Table 1).

Use of CAN-296 at concentrations of 8 and 16 fold greater than the MIC resulted in the killing of $99.6 \%$ and $99.9 \%$ of Candida albicans within 15 minutes of exposure, suggesting that CAN-296 is a rapidly acting fungicidal agent. The fungicidal activity of CAN-296 was more rapid than that of AMB in both Candida species.

The lack of marked activity of CAN-296 against A. fumigatus was in contrast to its impressive fungicidal activity demonstrated against Candida species. The reason for the relative resistance of $A$. fumigatus to CAN-296 is not clear. However, it is not unusual for an antibiotic that is highly active against one group of fungi to possess little activity against others ${ }^{12)}$.

In summary, CAN-296 demonstrates an excellent in vitro inhibitory antifungal and fungicidal spectrum.
CAN-296 was active against azole-resistant Candida. Coupled with its in vitro stability and rapidity of action, this complex carbohydrate makes an excellent candidate for a next generation therapeutic agent. Further investigations of its in vivo activity and toxicity are warranted.

\section{References}

1) Polak, A. \& P. G. Hartman: Antifungal chemotherapyare we winning? Prog. Drug Res. 37: 131 269, 1991

2) Gallis, H. A.; H. DreW \& W. W. Pickard: Amphotericin B: 30 years of experience. Rev. Inect. Dis. 12: 308 329, 1990

3) Baily, G. G.; F. M. Perry, D. W. Denning \& B. K. MANDEL: Fluconazole resistance candidosis in an HIV cohort. AIDS 8: 787 792, 1994

4) Komshian, S. V.; A. Uwaydah, J. D. Sobel \& L. R. Crane: Fungemia caused by Candida species and Torulopsis glabrata in hospitalized patients. Frequency, characteristics, and evaluation of factors influencing outcome. Rev. Infect. Dis. 11: 379 390, 1989

5) Nobre, G.; E. Mendes, M. Charrua \& O. Cruz: Ketoconazole resistance in Torulopsis glabrata. Mycopathologia 107: $51 \sim 55,1989$

6) VAZQUEZ, J. A.; M. LYNCH \& J. D. SOBEL: In vitro activity of a new pneumocandin antifungal agent, L-733,560, against azole-susceptible and resistant Candida and Torulopsis species. Antimicrob. Agents Chemother. 39: 2689 2691, 1995

7) Warnock, D.; J. Bruke, N. J. Cope, E. Johnson, N. von FRAUNHOFER \& E. WILLIAMS: Fluconazole resistance in Candida glabrata. Lancet I: 1310, 1988

8) Wingard, J. R.; W. G. Merz, M. G. Rinaldi, T. R. JOHNSON, J. E. KARP \& R. SARAL: Increase in Candida krusei infection among patients with bone marrow transplantation and neutropenia treated prophylactically 
with fluconazole. N. Engl. J. Med. 18: 1274 1277, 1991

9) National Committee for Clinical Laboratory Standards: Reference method for broth dilution antifungal susceptibility testing for yeast; Tentative standard. Document M27-T. National Committee for Clinical Laboratory Standards, Wayne, Pa, 1995

10) Espinel-Ingroff, A; K. Dawson, M. Pfaller, E. Anaissie, B. Breslin, D. Dixon, A. Fothergill, V. Paetznick, J. Peter, M. Rinaldi \& T. Walsh: Comparative and collaborative evaluation of standardization of antifungal susceptibility testing for filamentous fungi. Antimicro. Agents Chemother. 39: 314 319, 1995
11) Manavathu, E. K.; G. J. Alangaden \& S. A. Lerner: A comparative study of the micro- and macrobroth dilution techniques for the determination of in vitro susceptibility of Aspergillus fumigatus. In Abstract of the $95^{\text {th }}$ Annual Meeting of the American Society for Microbiology, Washington, DC. Abstract C356, 1995

12) Venkateswarlu, K.; D. W. Denning, N. J. Manning \& S. L. KELLY: Comparion of D0870, a new triazole antifungal agent, to fluconazole for inhibition of Candida albicans cytochrome P-450 by using in vitro assays. Antimicrob. Agents Chemother. 40: 1382 1386, 1996 